\title{
Binding energy of hydrogenic impurities in quantum dots under intense laser radiation
}

\author{
C. González-Santander ${ }^{1}$, T. Apostolova ${ }^{2}$ and F. \\ Domínguez-Adame ${ }^{1}$ \\ ${ }^{1}$ GISC, Departamento de Física de Materiales, Universidad Complutense, E-28040 \\ Madrid, Spain \\ ${ }^{2}$ Institute for Nuclear Research and Nuclear Energy, 72 Tzarigradsko chaussee Blvd, \\ 1784 Sofia, Bulgaria
}

E-mail: cglezsantander@fis.ucm.es

\begin{abstract}
.
We calculate the binding energy of on- and off-center hydrogenic impurities in a parabolic quantum dot subjected to an intense high-frequency laser field. An exactly solvable model is introduced for calculating the binding energy that replaces the actual Coulomb interaction with the donor by a nonlocal separable potential. The separable potential allows us to solve exactly the problem and all calculations are carried out analytically. The action of the laser irradiation results in dressed Coulomb and confinement potentials. At low laser intensity the binding energy is found to decrease when the impurity is shifted away from the origin. On the contrary, at high laser intensity and strong confinement the opposite behaviour is observed. We propose a simple one-dimensional model that explains the observed crossover.
\end{abstract}

PACS numbers: 73.22.Dj, 73.21.FG, 85.35.Be

Journal of Physics: Condensed Matter 25, 335802 (2013) 


\section{Introduction}

During the past several years, much experimental and theoretical work has been done on the binding energy of hydrogenic impurities located in quantum dots (QDs) and quantum rings [1-11]. The spatial confinement of carriers leads to a strong enhancement of the binding energy in these nanostructures, as compared to bulk semiconductors or even in quantum wells or quantum wires [12,13]. The enhancement of the binding energy can be detected by optical techniques since optical absorption lines in nanostructures are blue-shifted and carry information about the confinement of carriers. As a consequence, the blue-shift of the absorption lines makes spectroscopy tools very useful to characterize electron states bound to hydrogenic impurities in zero-dimensional nanostructures [14]. Most important, changes in the electronic and optical properties of nanostructures may be controlled by an appropriate selection of the sample geometry and material parameters, opening new potential applications in optoelectronics.

The use of optical techniques requires theoretical modeling of the influence of the laser field on the binding energy of carriers bound to hydrogenic impurities. Previously, Fanyao et al. calculated the binding energy of an on-center donor hydrogenic impurity in a quantum well [15] and in a QD [16] subjected to an intense high-frequency laser. The confinement of carriers in the QD was modeled by a spherical potential well with finite or infinite barrier. The intense laser field dresses the Coulomb potential and makes it dependent on the laser intensity [17-21]. A variational approach was used to calculate the binding energy of the on-center donor hydrogenic impurity. They predicted a fast decrease of the binding energy with increasing the laser intensity. Recently, Yesilgul et al. observed a similar behaviour for impurities in QD when the confining potential is also dressed by the laser radiation [22]. They analyzed the effect of shifting the impurity from the center to the middle of the QD, keeping the laser polarization parallel to the growth direction. Furthermore, in these works only the weak confinement regime was considered because the sizes of the QDs were larger than the effective Bohr radius. Therefore, a detailed study of the strong confinement regime and the effects of varying the laser polarization is needed.

In this work we present an alternative approach based on the non-local (separable) potential (NLP) method, in which the actual Coulomb potential is replaced by a projective operator $[23,24]$. The NLP method has already been successfully used to determine the binding energy of confined excitons in parabolic QDs in a closed form [25]. This method yields an exactly solvable envelope-function equation from which the electron states can be readily obtained with the desired accuracy. In addition, the NLP method can be generalized to describe a dressed Coulomb potential when a quantum wire is irradiated by an intense high-frequency laser field [26]. Using this generalized NLP method, an increase of the exciton energy with increasing the laser intensity was found [26]. The exciton energy reaches a saturation value at very high laser intensity, that is larger for small radius of the quantum wire. The NLP method is particularly useful when the confining potential is parabolic since the Green's function takes a simple 
form [25], although it can be applied to other models of confining potentials.

Our aim in this paper is to show that closed expressions for the binding energy of hydrogenic impurities can be obtained even if the system is subjected to an intense laser field. To this end we consider the laser effects on both Coulomb potential (replaced by a NLP) and QD confinement potential [15]. In addition, our approach is more general than the one presented by Fanyao et al. [16] and Yesilgul et al. [22] since it is valid for both on-center and off-center impurities and different laser polarizations. As a main result, we find a crossover of the binding energy as the donor is shifted from the origin: At low laser intensity the binding energy decreases but the opposite trend is observed under strong laser irradiation. A simple one-dimensional model is proposed to explain the observed trend.

\section{Theoretical model}

We consider a two-dimensional (2D) electron gas of noninteracting electrons confined in a semiconductor QD. In the framework of the effective-mass approximation [27], the single-electron Hamiltonian in the presence of a donor hydrogenic impurity can be written as $\mathcal{H}=\boldsymbol{p}^{2} / 2 m+V_{\mathrm{QD}}(\boldsymbol{r})+V_{\mathrm{d}}(\boldsymbol{r})$. The pair $\mathbf{p}$ and $\mathbf{r}$ are the usual momentum and coordinate in the plane of the 2D electron gas, respectively. The effective mass of the electron is denoted by $m$. Here $V_{\mathrm{QD}}(\boldsymbol{r})$ is the parabolic QD potential, assumed to be radially symmetric and independent of the polar angle. The Coulomb potential due to a hydrogenic donor impurity located at position $\boldsymbol{r}_{\mathrm{d}}$ from the origin and screened by the background dielectric constant $\epsilon$ is $V_{\mathrm{d}}(\boldsymbol{r})=-e^{2} / \epsilon\left|\boldsymbol{r}-\boldsymbol{r}_{\mathrm{d}}\right|$.

\subsection{Intense high-frequency laser field}

We now subject the system to the action of an intense laser field, represented by a linearly polarized plane wave of frequency $\Omega$. Under the dipole approximation the corresponding vector potential is $\boldsymbol{A}(t)=A_{0} \widehat{\boldsymbol{u}} \cos \Omega t$, where $\widehat{\boldsymbol{u}}$ is a unit vector that indicates the polarization. In order to obtain the electron-laser interaction potential, we follow the nonperturbative theory previously developed to describe the atomic states under intense high-frequency laser fields [17-21]. Applying the time dependent substitution $\boldsymbol{r} \rightarrow \boldsymbol{r}+\boldsymbol{\alpha}(t)$, the Schrödinger equation can be written as

$$
i \hbar \frac{\partial \psi(\boldsymbol{r}, t)}{\partial t}=\left[-\frac{\hbar^{2}}{2 m} \nabla^{2}+V_{\mathrm{QD}}(\boldsymbol{r}+\boldsymbol{\alpha}(t))+V_{\mathrm{d}}(\boldsymbol{r}+\boldsymbol{\alpha}(t))\right] \psi(\boldsymbol{r}, t),
$$

where $\boldsymbol{\alpha}(t)=-(e / m c) \int^{t} A\left(t^{\prime}\right) \mathrm{d} t^{\prime}=\boldsymbol{\alpha}_{0} \sin \Omega t$. Here $\boldsymbol{\alpha}_{0} \equiv-\left(e A_{0} / m \Omega c\right) \widehat{\boldsymbol{u}}$ represents the quiver motion of a classical electron in the laser field. The Schrödinger equation (1) can be cast in a set of coupled time-dependent differential equations for the Floquet components of the wave function. It could be solved by an iteration scheme but to the lowest order of the iteration, namely in the high-frequency limit, the system reduces to 
the time-independent Schrödinger equation [17]

$$
\left[\frac{\boldsymbol{p}^{2}}{2 m}+V_{\mathrm{QD}}\left(\boldsymbol{r}, \boldsymbol{\alpha}_{0}\right)+V_{\mathrm{d}}\left(\boldsymbol{r}, \boldsymbol{\alpha}_{0}\right)\right] \psi_{0}(\boldsymbol{r})=E \psi_{0}(\boldsymbol{r}),
$$

where $\psi_{0}$ is the zeroth Floquet component of the wave function and $E$ is the energy. $V\left(\boldsymbol{r}, \boldsymbol{\alpha}_{0}\right)$ is defined as the dressed potential that depends on the parameters of the laser field only through $\boldsymbol{\alpha}_{0}$. In Gaussian units, the dressing parameter $\alpha_{0} \equiv\left|\boldsymbol{\alpha}_{0}\right|$ is related to the time-averaged irradiance of the laser beam $I$, referred for simplicity to as intensity, as follows

$$
\alpha_{0}=\sqrt{\frac{8 \pi I}{c}} \frac{e}{m \Omega^{2}} .
$$

Under these circumstances, in the high-frequency regime, the electron only feels the static distorted potential $V\left(\boldsymbol{r}, \boldsymbol{\alpha}_{0}\right)$, the dressed potential associated with the original interaction potential $V(\boldsymbol{r})$. Actually this dressed potential is the time averaged over a period of the oscillating potential $V(\boldsymbol{r}, \boldsymbol{\alpha}(t))$, which is given by the integral [17]

$$
V\left(\boldsymbol{r}, \boldsymbol{\alpha}_{0}\right)=\frac{1}{\pi} \int_{-1}^{1} V\left(\boldsymbol{r}+\xi \boldsymbol{\alpha}_{0}\right)\left(1-\xi^{2}\right)^{-1 / 2} \mathrm{~d} \xi .
$$

Proceeding further, it has been shown that for the case of the Coulomb potential, its dressed counterpart can be reasonably well written as [19]

$$
V_{\mathrm{d}}\left(\boldsymbol{r}, \boldsymbol{\alpha}_{0}\right)=-\frac{e^{2}}{2 \epsilon}\left[\frac{1}{\left|\boldsymbol{r}-\boldsymbol{r}_{\mathrm{d}}-\boldsymbol{\alpha}_{0}\right|}+\frac{1}{\left|\boldsymbol{r}-\boldsymbol{r}_{\mathrm{d}}+\boldsymbol{\alpha}_{0}\right|}\right],
$$

and in the same way the confining potential

$$
V_{\mathrm{QD}}\left(\boldsymbol{r}, \alpha_{0}\right)=\frac{1}{4} m \omega^{2}\left[\left(\boldsymbol{r}+\boldsymbol{\alpha}_{0}\right)^{2}+\left(\boldsymbol{r}-\boldsymbol{\alpha}_{0}\right)^{2}\right],
$$

where we assume that the QD can be described by a parabolic potential with frequency $\omega$. These dressed potentials have been used as well in other studies of hydrogen impurities in quantum wires [15] and QD [16]. The single-electron Hamiltonian in the presence of the hydrogenic donor under the laser field becomes

$$
\mathcal{H}=\frac{\boldsymbol{p}^{2}}{2 m}+\frac{1}{2} m \omega^{2} \boldsymbol{r}^{2}+\frac{1}{2} m \omega^{2} \boldsymbol{\alpha}_{0}^{2}-\frac{e^{2}}{2 \epsilon}\left[\frac{1}{\left|\boldsymbol{r}-\boldsymbol{r}_{\mathrm{d}}-\boldsymbol{\alpha}_{0}\right|}+\frac{1}{\left|\boldsymbol{r}-\boldsymbol{r}_{\mathrm{d}}+\boldsymbol{\alpha}_{0}\right|}\right]
$$

Some considerations about the range of intensity values within which this approach is valid are in order. The first assumption of the present approach is that the laser field can be properly described under the dipole approximation. Therefore, the dressing parameter $\alpha_{0}$ should remain much smaller than the laser wavelength in order to satisfy this condition. This imposes an upper limit on the intensity values applicable to this study. In general, it is greater than the ones used in experimental setups, so it is not a real limit to our calculations. Nevertheless, the real condition for the intensity values comes from comparing $\alpha_{0}$ with the size of the bound system in the absence of the laser field, namely the effective Bohr radius, in 2D for the system under consideration, denoted as $a_{2 \mathrm{D}}$ hereafter. We impose as limiting values for intensity those that make $\alpha_{0} \sim a_{2 \mathrm{D}}$. Therefore, from (3) one obtains that the maximum intensity scales as 
$I_{\max } \sim a_{2 \mathrm{D}}^{2} \Omega^{4}[15,28]$. As an example, for a typical semiconductor such as GaAs and a laser of practical interest, such as $\mathrm{CO}_{2}\left(\Omega=2 \times 10^{14} \mathrm{~s}^{-1}\right)$, the model is applicable when the intensity $I$ is in the range $10^{7}-10^{12} \mathrm{~W} / \mathrm{cm}^{2}$, which is available in practice.

\subsection{Non-local separable potential}

The eigenfunctions of the corresponding Schrödinger equation (2) cannot be expressed in terms of elementary functions. From (5) it becomes apparent that under the laser field the system is equivalent to a single electron in a two-center Coulomb field confined in a parabolic QD. This analogy allows us to replace the effective Coulomb potentials in (5) by a pair of NLPs to obtain the envelope-function $|\chi\rangle$ from the effective-mass equation $[25,26]$

$$
\mathcal{H}|\chi\rangle \longrightarrow\left(\mathcal{H}_{0}+V_{\mathrm{NL}}\right)|\chi\rangle=E|\chi\rangle
$$

with $\mathcal{H}_{0}=\boldsymbol{p}^{2} / 2 m+V_{\mathrm{QD}}\left(\boldsymbol{r}, \alpha_{0}\right)$. The NLP is projective operator defined as

$$
V_{\mathrm{NL}} \equiv-\frac{g \hbar^{2}}{4 m}\left[\left|v_{+}\right\rangle\left\langle v_{+}|+| v_{-}\right\rangle\left\langle v_{-}\right|\right] .
$$

$v_{ \pm}$will be referred to as shape functions and they will be specified later, and $g$ is named coupling constant. Equation (8) can be solved exactly for any arbitrary NLP, provided the eigenstates of the $\mathcal{H}_{0}$ are known. We compute the envelope-function $|\chi\rangle$ as follows

$$
|\chi\rangle=-\left(\mathcal{H}_{0}-E\right)^{-1} V_{\mathrm{NL}}|\chi\rangle=\frac{g \hbar^{2}}{4 m} \sum_{\nu} \sum_{s= \pm} \frac{\left|\varphi_{\nu}\right\rangle\left\langle\varphi_{\nu}\right|}{E_{\nu}-E}\left|v_{s}\right\rangle\left\langle v_{s} \mid \chi\right\rangle,
$$

where $\left|\varphi_{\nu}\right\rangle$ denotes the eigenstates of $\mathcal{H}_{0}$ with eigenvalues $E_{\nu}$. Projecting onto the kets $\left|v_{ \pm}\right\rangle$and requiring the determinant of the resulting matrix to vanish we arrive at

$$
\left[S_{+}(E)-1\right]\left[S_{-}(E)-1\right]=|C(E)|^{2},
$$

where for brevity we have introduced the following definitions

$$
\begin{aligned}
& S_{ \pm}(E)=\frac{g \hbar^{2}}{4 m} \sum_{\nu} \frac{1}{E_{\nu}-E}\left|\left\langle v_{ \pm} \mid \varphi_{\nu}\right\rangle\right|^{2} \\
& C(E)=\frac{g \hbar^{2}}{4 m} \sum_{\nu} \frac{1}{E_{\nu}-E}\left\langle v_{+} \mid \varphi_{\nu}\right\rangle\left\langle\varphi_{\nu} \mid v_{-}\right\rangle .
\end{aligned}
$$

The transcendental equation (11a) provides the electron energy $E$ in the nanostructure in the presence of the laser field, for any arbitrary shape functions $v_{ \pm}$and coupling constant $g$.

The coupling constant $g$ is not an adjustable parameter of the model. This can be understood from the fact that we might obtain the energy level of an electron bound to the donor impurity in an infinite $2 \mathrm{D}$ semiconductor, $E_{2 D}=-4 \mathrm{Ry}^{*}$, when the confining potential is switched off $(\omega \rightarrow 0)$ and the nanostructure is not under laser irradiation $\left(\alpha_{0} \rightarrow 0\right)$. Here $\mathrm{Ry}^{*}$ is the effective Rydberg, which is the impurity binding energy in a three-dimensional bulk semiconductor. Therefore, the resulting value of the coupling constant depends on the shape functions chosen and the dimensionality of the system under study. We take a Gaussian shape function which was formerly used for studying 
hydrogenic impurities located at position $\boldsymbol{r}_{\mathrm{d}}$ in QD $[10,11]$. The dressing effect of the laser field on the Coulomb potential is included in the shape functions through a displacement $\pm \alpha_{0}$ as follows

$$
v_{ \pm}(\boldsymbol{r})=\frac{1}{\pi a^{2}} \exp \left(-\frac{\left(\boldsymbol{r}-\boldsymbol{r}_{\mathrm{d}} \pm \boldsymbol{\alpha}_{0}\right)^{2}}{a^{2}}\right),
$$

where $a$ is a free parameter that must be set properly to account for the ground state of the hydrogenic impurity in an infinite $2 \mathrm{D}$ semiconductor.

The calculation of the coupling constant $g$ is easily carried out in momentum space when $\omega=0$ and $\alpha_{0}=0$. Due to the translational invariance of $\mathcal{H}_{0}$ in this case, we can set $\boldsymbol{r}_{\mathrm{d}}=0$ without loss of generality. The Hamiltonian reduces to $\mathcal{H}_{0}=\boldsymbol{p}^{2} / 2 \mathrm{~m}$ and its eigenstates are plane waves with momentum $\boldsymbol{p}$ and energy $E_{\boldsymbol{p}}=\boldsymbol{p}^{2} / 2 \mathrm{~m}$. Thus, the energy spectrum is continuous and the summations appearing in (11b) is replaced by an integration in momentum space. The shape function (12) simplifies to the one proposed in [10] and finally (11a) reduces to a much simpler transcendental equation. The coupling constant can be calculated easily from the Fourier transform of the shape function. Performing the same steps as in [10], the coupling constant is found to be

$$
g=\frac{4 \pi e^{-\mu / 2}}{\Gamma(0, \mu / 2)},
$$

where $\Gamma(b, z)$ is the incomplete Gamma function [29] and $\mu=\left(a / a_{2 \mathrm{D}}\right)^{2}$ is an adjustable parameter. This should be as small as possible in the numerical calculation so that the Gaussian function approaches the $\delta$-function limit.

\subsection{Donor bound electron in a parabolic quantum dot}

After having obtained the coupling constant, we now turn to the normalized eigenfunctions of $\mathcal{H}_{0}$, needed in order to find the energy from (11a) when the laser field is switched on and the impurity is located at an arbitrary position $\boldsymbol{r}_{\mathrm{d}}$. From (7) we notice that the eigenfunctions of $\mathcal{H}_{0}$ are the ones of an electron in a parabolic potential plus a constant potential $m \omega^{2} \alpha_{0}^{2} / 2$

$$
\varphi_{n \ell}(r, \theta)=R_{n \ell}(r) \frac{e^{i \ell \theta}}{\sqrt{2 \pi}},
$$

with quantum numbers $\ell=0, \pm 1, \pm 2 \ldots$ and $n=0,1,2, \ldots$ The radial function is given by $[30]$

$$
R_{n \ell}(r)=\sqrt{\frac{2 n !}{(n+|\ell|) !}} \frac{r^{|\ell|}}{\mathcal{L}^{|\ell|+1}} e^{-r^{2} / 2 \mathcal{L}^{2}} L_{n}^{|\ell|}\left(r^{2} / \mathcal{L}^{2}\right),
$$

where $\mathcal{L}=\sqrt{\hbar / m \omega}$ is the $\mathrm{QD}$ size and $L_{n}^{|\ell|}$ denotes the generalized Laguerre polynomial [29]. The eigenvalues are $E_{n \ell}=\hbar \omega(2 n+|\ell|+1)+m \omega^{2} \alpha_{0}^{2} / 2$.

We set $\boldsymbol{r}_{\mathrm{d}}$ along the $X$ axis without loss of generality. The overlap between the shape functions $v_{ \pm}$defined by (12) and the eigenfunctions $\varphi_{n \ell}$ is given by

$$
\left\langle v_{ \pm} \mid \varphi_{n \ell}\right\rangle=\frac{\sqrt{2 \pi}}{\pi a^{2}} \exp \left[\mp i \ell \lambda_{ \pm}-\frac{1}{a^{2}}\left(r_{\mathrm{d}}^{2}+\alpha_{0}^{2} \mp 2 r_{\mathrm{d}} \alpha_{0} \cos \phi\right)\right]
$$


Hydrogenic impurities in quantum dots under intense laser radiation

$$
\times \int_{0}^{\infty} d r r e^{r^{2} / a^{2}} R_{n \ell}(r) I_{|\ell|}\left[\sqrt{1+\delta_{ \pm}^{2}}\left(\frac{2 r r_{\mathrm{d}} \mp 2 r \alpha_{0} \cos \phi}{a^{2}}\right)\right],
$$

where $\phi$ is the angle between $\boldsymbol{\alpha}_{0}$ and the $X$ axis, namely the polarization angle of the laser radiation. The following parameters are defined in order to simplify the notation $\delta_{ \pm}=\alpha_{0} \sin \phi /\left(r_{\mathrm{d}} \mp \alpha_{0} \cos \phi\right)$ and $\lambda_{ \pm}=\arctan \delta_{ \pm} . I_{|\ell|}$ is the modified Bessel function [29]. After some algebra we obtain

$$
\begin{aligned}
& \left\langle v_{ \pm} \mid \varphi_{n \ell}\right\rangle=\frac{\sqrt{2 \pi}}{\pi a^{2}} \kappa_{n \ell} \exp \left(\mp i \ell \lambda_{ \pm}-\frac{z_{\mathrm{d}}^{2}}{2 \mu \beta}-\frac{\gamma^{2}}{\mu} \pm \frac{2 z_{\mathrm{d}} \gamma \cos \phi}{\mu \sqrt{2 \beta}}\right) \\
& \times \exp \left(\frac{\mu \beta \rho_{ \pm}^{2}\left(1+\delta_{ \pm}^{2}\right)}{2(1+\mu \beta)}\right) L_{n}^{|\ell|}\left(\frac{\mu^{2} \beta^{2} \rho_{ \pm}^{2}\left(1+\delta_{ \pm}^{2}\right)}{1-\mu^{2} \beta^{2}}\right),
\end{aligned}
$$

with

$$
\begin{aligned}
& \rho_{ \pm}=\frac{z_{\mathrm{d}}}{\mu \beta} \mp \frac{2 \gamma \cos \phi}{\mu \sqrt{2 \beta}} \\
& \kappa_{n \ell}=\sqrt{\frac{n !}{(n+|\ell|) !}}\left(\frac{1-\mu \beta}{1+\mu \beta}\right)^{n}\left(1+\frac{1}{\mu \beta}\right)^{-|\ell|-1} \rho_{ \pm}^{|\ell|}\left(1+\delta_{ \pm}^{2}\right)^{|\ell| / 2} .
\end{aligned}
$$

For brevity we introduced the confining parameter $\beta \equiv a_{2 \mathrm{D}}^{2} / 2 \mathcal{L}^{2}$. This parameter determines the magnitude of the donor-bound electron confinement in the QD since the larger $\beta$, the higher the confinement. The parameter $\gamma \equiv \alpha_{0} / a_{2 \mathrm{D}}$ gives the ratio between the laser intensity, parameterized by $\alpha_{0}$, and the Coulomb interactions defined by the Bohr radius, $a_{2 \mathrm{D}}$. In this study we will restrict ourselves to the range $0 \leq \gamma \leq 1$, which has been commonly used in the literature [16,22]. The position of the impurity will be measured in units of the size of the QD, $z_{\mathrm{d}}=r_{\mathrm{d}} / \mathcal{L}$.

Inserting (17) in (11a) we obtain a transcendental equation that it is solved numerically with minimal computational effort for any chosen set of parameters. In order to compare the results for different confinement strength, impurity position and laser intensity, we focus on the binding energy of the ground state, $E_{B}=m \omega^{2} \alpha_{0}^{2} / 2+\hbar \omega-E$.

\section{Results}

We present our results for different values of the confining parameter $\beta=a_{2 \mathrm{D}}^{2} / 2 \mathcal{L}^{2}$ and the parameter $\gamma=\alpha_{0} / a_{2 \mathrm{D}}$. Energy will be measured in units of the $2 \mathrm{D}$ effective Rydberg $4 \mathrm{Ry}^{*}$. In Ref. [10] it has been shown that good accuracy is achieved with $\mu=0.01$ in the coupling constant (13) and hereafter we take this value.

Figure 1 shows the binding energy of the ground state as a function of the parameter $\gamma=\alpha_{0} / a_{2 \mathrm{D}}$ for polarization angle $\phi=0$ and for three sizes $\mathcal{L}$. From top to bottom $\mathcal{L}=2 a_{2 \mathrm{D}}, \mathcal{L}=a_{2 \mathrm{D}}$ and $\mathcal{L}=a_{2 \mathrm{D}} / 2$, which allows us to study the full range from weak $\left(\mathcal{L}>a_{2 \mathrm{D}}\right)$ to strong $\left(\mathcal{L}<a_{2 \mathrm{D}}\right)$ confinement regimes. The curves correspond to different values of the impurity position, which is continuously shifted from the center to the edge of the QD. Different trends in the dependence of the binding energy on the laser intensity and the QD size are observed and discussed below. 
Hydrogenic impurities in quantum dots under intense laser radiation
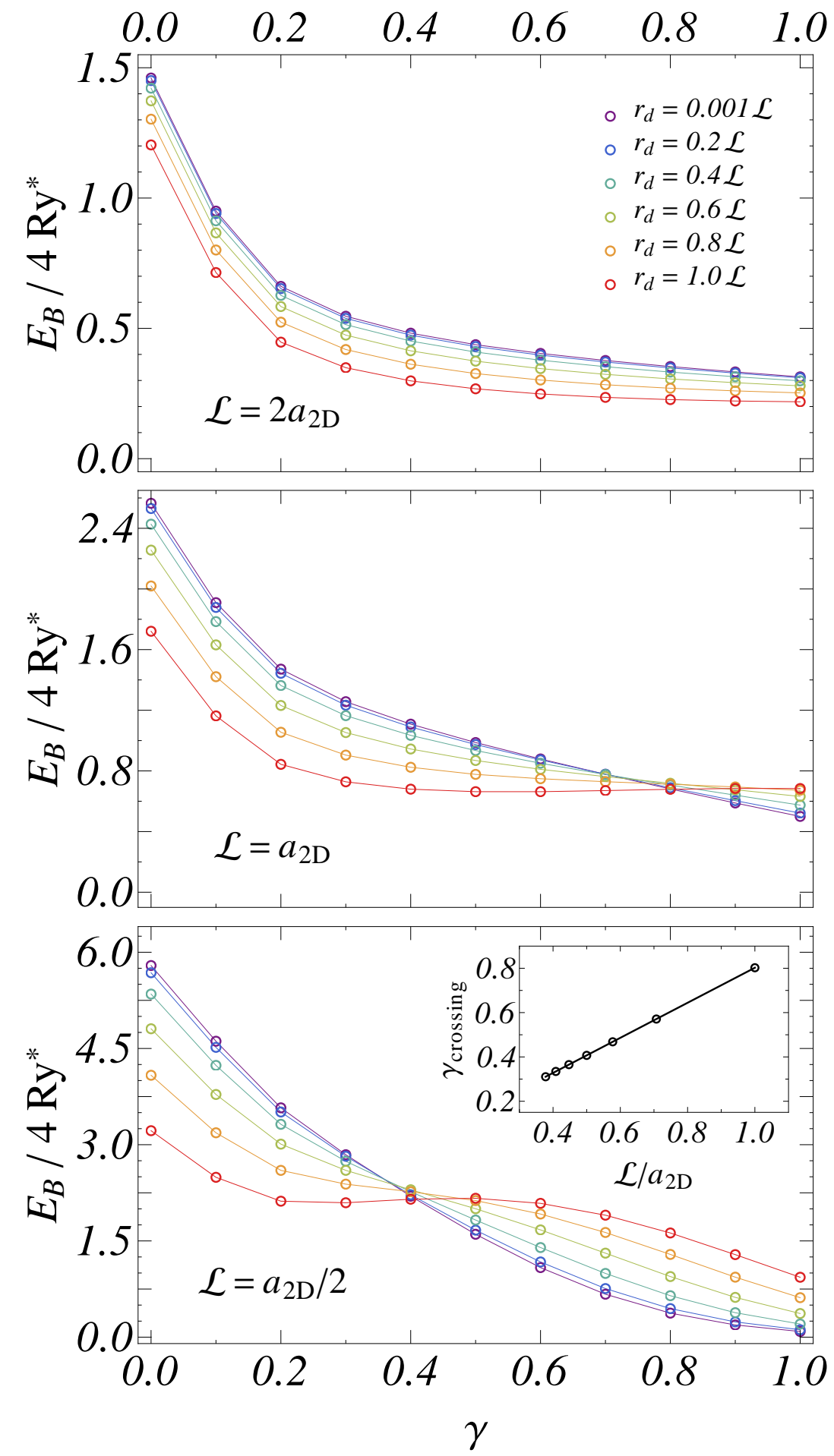

Figure 1. Binding energy in units of the 2D effective Rydberg as a function of the parameter $\gamma=\alpha_{0} / a_{2 \mathrm{D}}$ for three values of the QD size, indicated on each plot, and different impurity positions $r_{\mathrm{d}}$. The polarization angle is $\phi=0$. The inset shows the linear dependence of the value of $\gamma$ at the crossing on the QD size.

Binding energy when the laser is switched off. We introduce a much simpler onedimensional approach to explain qualitatively the decrease of the binding energy when 
the impurity is slightly shifted from the origin and $\gamma=0$, observed in figure 1 . It should be mentioned that similar behaviour was reported previously in Refs. [16,22] for different confinement potentials and theoretical approaches. The simplified model considers an electron moving in one-dimension and replaces the Coulomb potential by a $\delta$-function. The Hamiltonian reads $\mathcal{H}=\mathcal{H}_{0}-\left(e^{2} / \epsilon\right) \delta\left(x-x_{d}\right)$, where $\mathcal{H}_{0}=p^{2} / 2 m+(1 / 2) m \omega^{2} x^{2}$ is the Hamiltonian of a harmonic oscillator. Introducing the Green's function of the harmonic oscillator, it is a matter of simple algebra to arrive at the following equation for the energy levels $G_{0}\left(x_{d}, x_{d} ; E\right)=\epsilon / e^{2}$, namely

$$
G_{0}\left(x_{d}, x_{d} ; E\right)=\sum_{n=0}^{\infty} \frac{\left|\psi_{n}\left(x_{d}\right)\right|^{2}}{E_{n}-E}=\frac{\epsilon}{e^{2}}, \quad E<E_{n},
$$

where the sum runs over the eigenstates of the harmonic oscillator. The largest contribution to the sum in (18) comes from the ground state of the unperturbed oscillator $n=0$. Taking into account that $E_{0}-E=E_{B}$, equation (18) can be approximated as $\left|\psi_{0}\left(x_{d}\right)\right|^{2} / E_{B}=\epsilon / e^{2}$. When the impurity is slightly shifted from the origin the probability density $\left|\psi_{0}\left(x_{d}\right)\right|^{2}$ decreases and consequently $E_{B}$ must decrease too, as observed in figure 1.

Binding energy when the laser intensity is switched on. Figure 1 shows that $E_{B}$ decreases when $\gamma$ is not large (namely $\alpha_{0}<\mathcal{L}$ ), no matter the impurity position. However, when the confinement is enhanced and the size of the QD is of the order of the Bohr radius or even smaller, the curves show a well defined crossing in the range $0 \leq \gamma \leq 1$. As the impurity approaches the edge of the QD, the binding energy around the crossing point even increases with increasing $\gamma$. The crossing of the curves appears when the magnitude of the laser intensity is such that $\alpha_{0}$ is of the order of the QD size $\mathcal{L}$. Using the condition $\alpha_{0}=\mathcal{L}$ we can obtain an estimation of the value of the parameter $\gamma$ at the crossing $\gamma_{\text {crossing }}=1 / \sqrt{2 \beta}=\mathcal{L} / a_{2 \mathrm{D}}$. The inset of figure 1 shows this magnitude calculated from the crossing point of the curves for $r_{d}=0.001 \mathcal{L}$ and $r_{d}=1.0 \mathcal{L}$ for different $\mathcal{L} / a_{2 \mathrm{D}}$. As expected, a linear dependence of the crossing on the QD size is observed.

In order to explain this result we can proceed in the same line as before. Now the one-dimensional Hamiltonian reads $\mathcal{H}=\mathcal{H}_{0}-\left(e^{2} / 2 \epsilon\right)\left[\delta\left(x-x_{d}-\alpha_{0}\right)+\delta\left(x-x_{d}+\alpha_{0}\right)\right]$ with $\mathcal{H}_{0}=p^{2} / 2 m+(1 / 2) m \omega^{2}\left(x^{2}+\alpha_{0}^{2}\right)$. The resulting eigenvalue equation is

$$
\begin{aligned}
& \left(G_{++}\left(x_{d}, x_{d} ; E\right)-\frac{2 \epsilon}{e^{2}}\right)\left(G_{--}\left(x_{d}, x_{d} ; E\right)-\frac{2 \epsilon}{e^{2}}\right) \\
& =G_{+-}\left(x_{d}, x_{d} ; E\right) G_{-+}\left(x_{d}, x_{d} ; E\right),
\end{aligned}
$$

where

$$
G_{ \pm \pm}\left(x_{d}, x_{d} ; E\right)=\sum_{n=0}^{\infty} \frac{\psi_{n}\left(x_{d} \pm \alpha_{0}\right) \psi_{n}^{*}\left(x_{d} \pm \alpha_{0}\right)}{E_{n}-E}, \quad E<E_{n} .
$$

Considering only the contribution of the ground state to the sum (19b), the binding energy is found to be $E_{B}=E_{0}-E \sim\left|\psi_{0}\left(x_{d}+\alpha_{0}\right)\right|^{2}+\left|\psi_{0}\left(x_{d}-\alpha_{0}\right)\right|^{2}$, namely

$$
E_{B} \sim \exp \left(-\frac{\left(x_{d}+\alpha_{0}\right)^{2}}{\mathcal{L}^{2}}\right)+\exp \left(-\frac{\left(x_{d}-\alpha_{0}\right)^{2}}{\mathcal{L}^{2}}\right) \text {. }
$$


As explained before, from this simplified one-dimensional model a decrease in the binding energy when the laser is switched off $\left(\alpha_{0}=0\right)$ is predicted. It can be also observed that when the impurity is located at the origin $\left(x_{d}=0\right)$ a decrease of $E_{B}$ is also expected, in agreement to what is observed in figure 1. Nevertheless, when the laser intensity and the impurity position are modified simultaneously, two clear regimes are observed. At low laser irradiation $\alpha_{0}<\mathcal{L}$, equation $(19 c)$ predicts that the binding energy decreases after shifting the impurity from the origin or increasing the laser irradiation. This prediction is in perfect agreement to the results shown in figure 1 (see the curves of the lower panel at the left of the crossing point). On the other side, when $\alpha_{0}>\mathcal{L}$, an increase of the binding energy after increasing $x_{d}$ is deduced from $(19 c)$, in agreement to the results shown in figure 1 (see the curves of the lower panel at the right of the crossing point). Then, we are led to the conclusion that the one-dimensional model renders the behaviour observed in figure 1 even in the presence of the laser irradiation.

The binding energy also depends on the angle between the polarization direction and the impurity position $\boldsymbol{r}_{\mathrm{d}}$. Figure 2 shows the binding energy as a function of the parameter $\gamma$ in the strong confinement regime $\left(\mathcal{L}=a_{2 \mathrm{D}} / 2\right)$ and two different angles, $\phi=\pi / 4$ and $\phi=\pi / 2$. Results should be compared to those shown in the bottom panel of figure 1 , corresponding to $\phi=0$. When the impurity is close to the center of the QD $\left(r_{\mathrm{d}} \ll \mathcal{L}\right)$, the binding energy is almost independent of the polarization angle, as expected. However, the binding energy is strongly influenced by the polarization angle when the impurity is located close to the edge of the QD $\left(r_{\mathrm{d}} \simeq \mathcal{L}\right)$. As a result, the crossing appears at higher values of the parameter $\gamma$. This shift can be easily understood by noting that the projection of the vector $\boldsymbol{\alpha}_{0}$ on the $X$ axis is $\alpha_{0} \cos \phi$. Therefore, the laser intensity must be increased by a factor $1 / \cos \phi$ to compensate it. In other words, the crossing scales as $\gamma_{\text {crossing }}(\phi) \sim 1 / \cos \phi$, as seen in the inset of figure 2 .

In order to experimentally observe the crossing effect, the QDs should be based on a semiconducting material such that the effective 2D Bohr radius is of the order of the actual achievable QDs sizes. A good candidate is InSb/GaSb, for which $a_{2 \mathrm{D}} \sim 30 \mathrm{~nm}$. QDs based on InSb/GaSb with lateral sizes in the range 20 - 30 nm [33] or even smaller, of the order of $10 \mathrm{~nm}$ [34], have been grown by molecular-beam epitaxy. As we pointed out before, the intensity values for which our approach is valid should be in the range $10^{7}-10^{12} \mathrm{~W} / \mathrm{cm}^{2}$ for commonly used laser frequencies. Therefore, as long as these

values are used, the crossing effect should be detected experimentally. In the case of semiconductors with smaller effective 2D Bohr radii, the crossing should be observed at higher values of intensity.

\section{Conclusions}

We have introduced an exactly solvable model from which the binding energy of on-center and off-center hydrogenic impurities in a QD under high-frequency laser irradiation can be obtained. In the framework of the effective-mass approximation, 
Hydrogenic impurities in quantum dots under intense laser radiation

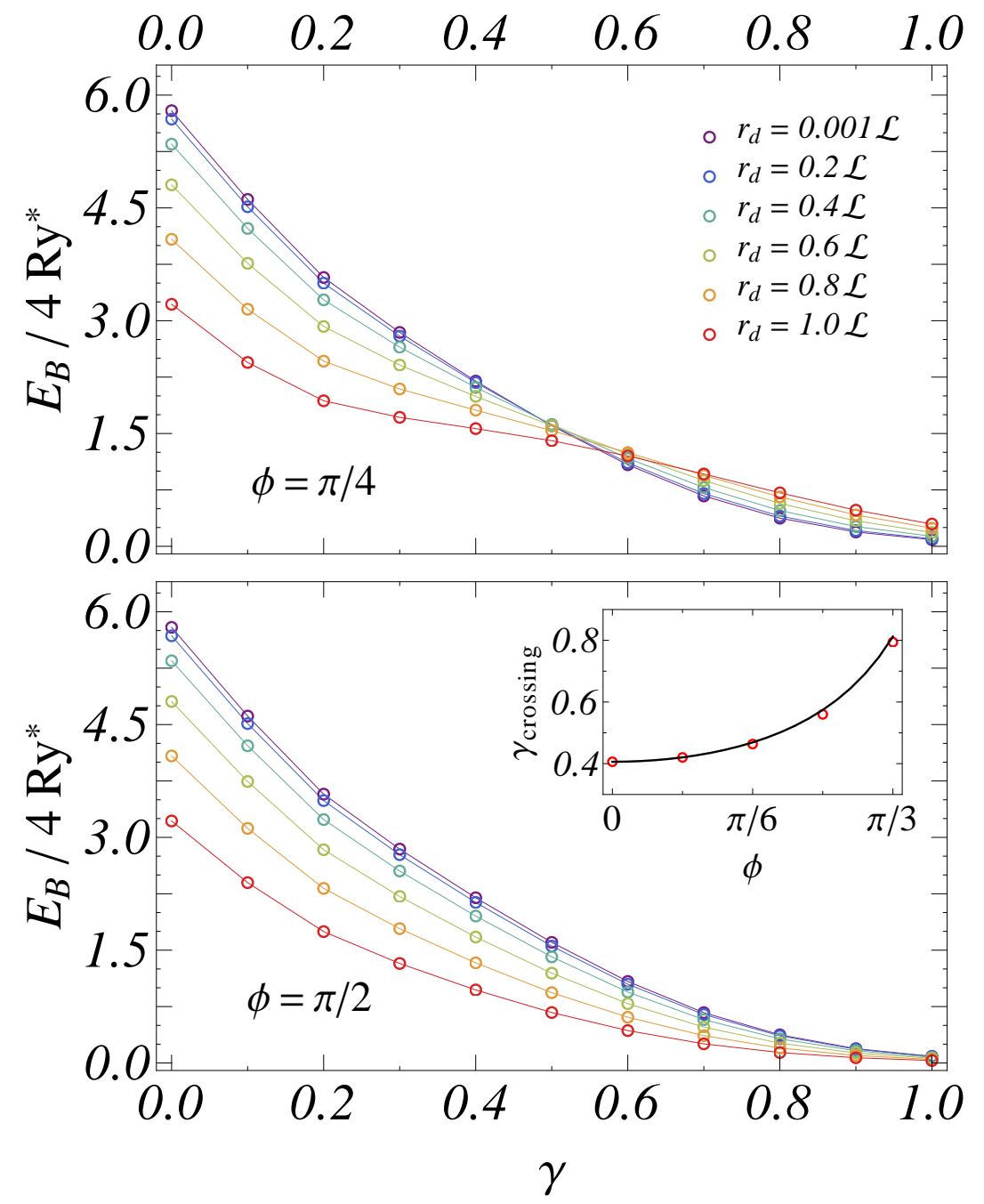

Figure 2. Binding energy in units of the 2D effective Rydberg as a function of the parameter $\gamma=\alpha_{0} / a_{2 \mathrm{D}}$ for two values of the polarization angle, indicated on each plot, and different impurity positions $r_{\mathrm{d}}$. The QD size is $\mathcal{L}=a_{2 \mathrm{D}} / 2$. The inset compares the value of $\gamma$ at the crossing (open circles) with the theoretical prediction $\gamma_{\text {crossing }}(\phi)=\gamma_{\text {crossing }}(0) / \cos \phi($ solid line $)$.

the confining potential arising from the QD is assumed to be parabolic with confining frequency $\omega$. Our study is based on the NLP approach, in which the dressed Coulomb interaction between the electron and the donor is replaced by a projective operator. We have taken Gaussian NLP, which was found to be suitable for describing off-center hydrogenic impurities in QDs [10,11]. As a major result, we have shown that the binding energy is found to decrease as the impurity moves away from the center under low irradiation intensity. However, the opposite trend is observed under strong irradiation. The transition between these two regimes occurs when the laser-dressing parameter equals the size of the QD. Finally, for elucidating the physics underlying the effect observed, we have introduced a simplified one-dimensional model that captures the relevant regimes observed in our calculations. 


\section{Acknowledgments}

Work at Madrid was supported by MICINN (project MAT2010-17180). C. G.-S. acknowledges financial support from Comunidad de Madrid and European Social Fundation. T.A. was sponsored by the Air Force Office of Scientific Research, Air Force Material Command, USAF, under grant number FA8655-12-1-2052.

\section{References}

[1] Zhu J-L, Zhao J-H, Duan W-H and Gu B-L 1992 Phys. Rev. B 467546.

[2] Porras-Montenegro N and Pérez-Merchancano S T 1992 Phys. Rev. B 469780.

[3] Porras-Montenegro N, Pérez-Merchancano S T and Latgé A 1993 J. Appl. Phys. 747624.

[4] Ribeiro F J and Latgé A 1994 Phys. Rev. B 504913.

[5] Zhu J-L, Zhao J-H and Xiong J-J 1994 J. Phys.: Condens. Matter 65097.

[6] Bose C 1998 J. Appl. Phys. 833089.

[7] Hsieh C-Y and Chuu D-S 2000 J. Phys.: Condens. Matter 128641.

[8] Zaratiegui J, Pietiläinen P and Hyvönen P 2002 Phys. Rev. B 66195324.

[9] García L F, Marín J H and Mikhailov I D Brazilian 2006 J. Phys. 36878.

[10] Lima R P A, Amado M and Domínguez-Adame F 2008 Nanotechnology 19135402.

[11] Lima R P A and Amado M 2008 J. Lumin. 128858.

[12] Bastard G 1981 Phys. Rev. B 244714.

[13] Bastard G 1982 Surf. Sci. 113165.

[14] Morgan G P, Ogawa K, Hiruma K, Kakibayashi H and Katsuyama T 1991 Solid State Commun. 80235.

[15] Fanyao Q, Fonseca A L and Nunes O A C 1996 Phys. Rev. B 5416405.

[16] Fanyao Q, Fonseca A L and Nunes O A C 1997 J. App. Phys. 821236.

[17] Gavrila M and Kaminski J Z 1984 Phys. Rev. Lett. 52613.

[18] Pont M, Walet N R, Gavrila M and McCurdy C W 1988 Phys. Rev. Lett. 61939.

[19] Ehlotzky F 1988 Phys. Lett. A 126524.

[20] Kramers H 1956 Collected Scientific Papers (North-Holland, Amsterdam) p 866.

[21] Henneberger W C 1968 Phys. Rev. Lett. 21838.

[22] Yesigul U, Sakiroglu S, Kasapoglu E, Sari H and Sokmen I 2011 Physica B 4061441.

[23] Knight B W and Peterson G A 1963 Phys. Rev. 1321085.

[24] Sievert P R and Glasser M L 1973 Phys. Rev. B 71265.

[25] López S and Domínguez-Adame F 2002 Semicond. Sci. Technol. 17227.

[26] González-Santander C and Domínguez-Adame F 2010 Physics Letters A 3742259.

[27] Davies J H 1998 The Physics of Low-Dimensional Semiconductors (Cambridge University Press, Cambridge).

[28] Lima C A S and Miranda L C M 1981 Phys. Rev. A 233335.

[29] Abramowitz M and Stegun I 1972 Handbook of Mathematical Functions (Dover, New York).

[30] Chruściński D 2006 Ann. Phys. 321840.

[31] Marinescu M and Gavrila M 1995 Phys. Rev. A 532513.

[32] Sari H, Kasapoglu E, Sökmen I and Güneş M 2003 Phys. Lett. A 319211.

[33] Tasco V, Deguffroy N, Baranov A N, Tournié E, Satpati B, Trampert A, Dunaevskii M S and Titkov A 2006 Appl. Phys. Lett. 89263118.

[34] Deguffroy N, Tasco V, Baranov A N, Tournié E, Satpati B, Trampert A, Dunaevskii M S, Titkov A and Ramonda M 2007 J. Appl. Phys. 101124309. 